\title{
A study of angiographic severity in patients with coronary artery disease and hyperuricemia
}

\author{
Subhraprakash Pramanik ${ }^{1}$, Koushik Mandal ${ }^{2}$, Arpan Kumar Dey ${ }^{3}$, Pijush Kanti Mandal ${ }^{4}$, \\ Shovan Kumar Das ${ }^{5}$, Tanmoy W Momin ${ }^{6}$, Mrinmoy Mitra ${ }^{7}$, Monojit Mondal ${ }^{8}$, Satyendra Nath Dutta ${ }^{9}$ \\ ${ }^{1}$ RMO Cum Clinical Tutor, Gen. Medicine, Midnapore Medical College, Paschim Midnapore, West Bengal, 2Senior Resident, Cardiology, \\ Burdwan Medical College (SSWH), Burdwan, West Bengal, ${ }^{3}$ Consultant Physician, Jalpayguri, West Bengal, ${ }^{4}$ Assistant Professor, General \\ Medicine, College of Medicine and Sagore Dutta Hospital, Kolkata, ${ }^{5}$ Specialist Physician, Ghatal SD Hospital, Paschim Midnapore, West \\ Bengal, ${ }^{6}$ Consultant Physician, Tura, Meghalaya, ${ }^{7}$ RMO Cum Clinical Tutor, Pulmonary Medicine, Calcutta National Medical College, Kolkata, \\ ${ }^{8}$ Senior Resident, Paedictrics, Institute of Child Health, Kolkata, ${ }^{9}$ Post-Doctoral trainee, Cardiology, Medical College, Kolkata
}

Backgrounds: Hyperuricemia has not yet been established as cardiovascular risk factor. We aimed to study the angiographic severity in patients with coronary artery disease (CAD) and hyperuricemia. Materials and Methods: In this observational cross-sectional study we measured serum uric acid level in 82 patients of CAD who underwent coronary angiography in catheterisation laboratory of our Institution. Severity of CAD was determined on the basis of Gensini score, number of diseased vessels, critical lesions and total occlusions. Results: The prevalence of hyperuricemia in patients with CAD was $42.68 \%$ in our study. Hyperuricemia was associated with higher Gensini score (33.33 \pm 10.64 vs. $22.90 \pm 6.75$, p value $<0.001$ ), number of critical lesions $(1.03 \pm 0.84$ vs. $0.63 \pm 0.72$, p value 0.031$)$, total occlusions $(0.67 \pm 0.47$ vs. $0.37 \pm 0.48$, p value 0.007$)$ and dyslipidemia $(63.6 \%$ vs. $30.6 \%$, p value 0.003 ) more frequently compared to normouricemic patients. And also the higher serum uric acid level was correlated with higher Gensini score (beta $0.418, t 4.430$, p value $<0.001$, $95 \% \mathrm{Cl} 0.036$ and 0.094 ) and frequent number of total occlusion (beta $0.338, \mathrm{t} 3.589$, $\mathrm{p}$ value $0.001,95 \% \mathrm{Cl} 0.462$ and 1.613). Conclusion: Hyperuricemia was associated with higher Gensini score, frequent total occlusions and critical lesions in patients with Coronary Artery Disease compared to patients with normal uric acid level and also it was significantly correlated with higher Gensini score and frequent total occlusions.

Key words: Hyperuricemia, Angiographic severity, Gensini score, Coronary artery disease (CAD)

\section{INDRODUCTION}

Hyperuricemia induces endothelial dysfunction (ED), ${ }^{1}$ the main mechanism by which it promotes atherosclerosis. Kim SY et al observed in their study, an association of increased serum uric acid with an increased risk of CHD incidence and mortality. ${ }^{2} \mathrm{~A}$ Russian study reported that the patients with arterial hypertension of the first-second degree with persistent elevated levels of uric acid in blood serum had significantly higher levels of ED markers: albuminuria, plasmic endothelin concentration. ${ }^{3}$ Hyperuricemia also predicts adverse outcomes in patients with heart failure. ${ }^{4}$ Hyperuricemia also associated with various components of the Insulin Resistance Syndrome. ${ }^{5}$ In this study we aimed to correlate the angiographic severity in patients with coronary artery disease (CAD) with high serum uric acid levels.

\section{MATERIALS AND METHODS}

In this observational cross-sectional study we measured serum uric acid level in 82 patients of CAD who underwent coronary angiography in catheterisation laboratory of our Institution from May 2013 to December 2014. Informed written consent was taken from every subjects and our study protocol was approved by Institutional Ethics Committee of Burdwan Medical College at the commencement of study. Severity of CAD was determined on the basis of Gensini 
score, number of diseased vessels, critical lesions and total occlusions. Demographic and clinical profile and laboratory data were collected for every patient. Cardiovascular risk factors were recorded according to standard definitions. Patients with history of IHD, heart failure, liver and kidney diseases, haematological or oncological disorders and chronic infections, taking diuretics, multivitamins, alcohol and on drugs interfering with serum uric acid levels were excluded from this study. Serum uric acid level was done after $>8$ hours of fasting and analysis was done by photometric technique with normal reference range of $3.5-6.5 \mathrm{mg} / \mathrm{dl}$. Coronary angiography was performed through right femoral or right radial route by using Judkin's technique and on Bio-core (Siemens) and Integrus (Philips) machines in catheterization laboratory of Super-Speciality Wing Hospital of Burdwan Medical College in West Bengal, India, by experienced cardiologist unaware of clinical or biochemical profile of patients. The Gensini score was used to evaluate the severity of atherosclerotic lesions on angiogram. The Gensini score was computed by assigning a severity score to each coronary stenosis according to the degree of luminal narrowing (visual assessment) (Table 1). This number is then multiplied by a factor that takes into account the geographical importance of the lesion position in the coronary arterial tree (Table 2). The Gensini score was then expressed as sum of scores of all coronary arteries. Critical lesion was defined as $\geq 70 \%$ stenosis involving proximal part of any of the three main coronary arteries or $\geq 50 \%$ of left main stem stenosis. Total occlusion was defined as 100\% occlusion with no ante grade flow of contrast distal to the lesion. A vessel was counted as diseased vessel if it has $\geq 50 \%$ stenosis at any level. Statistical Analysis was performed using SPSS V version 20 for windows with appropriate methods. A 2-tailed P value $<0.05$ was considered statistically significant.

\section{RESULTS}

The prevalence of hyperuricemia in patients with CAD in this study was $42.68 \%$ ( 35 out of total 82 patients). The mean serum uric acid level was high in hyperuricemic group (serum uric acid $>6.5 \mathrm{mg} / \mathrm{dL}$ ) compared to normouricemic (serum uric acid $<6.5 \mathrm{mg} / \mathrm{dL}$ ) group $(7.59 \pm 8.12 \mathrm{mg} / \mathrm{dL}$ vs. $4.83 \pm 0.66 \mathrm{mg} / \mathrm{dL}$, p value $<0.001$ ) (Table 3 ). There were no significant difference between two groups regarding mean age ( $53.76 \pm 6.28$ years vs. $53.45 \pm 6.25$ years, $p$ value 0.828 ), gender distribution (Male: Female, 63.6\%:36.4\% vs. $59.2 \%: 40.8 \%$, p value 0.685$)$, positive family history of heart disease (39.4\% vs. $38.8 \%$, p value 0.955$)$, smoker ( $24.2 \%$ vs. $34.7 \%$, p value 0.313$)$, hypertensive (66.7\% vs. $49.0 \%$, p value 0.113$)$, diabetes ( $33.3 \%$ vs. $24.5 \%$, $\mathrm{p}$ value 0.382 ), obesity ( $51.5 \%$ vs. $44.9 \%$, p value 0.556$)$, STEMI $(57.6 \%$ vs. $57.1 \%$, p value 0.969$)$, NSTEMI $(27.3 \%$ vs. $30.6 \%$, p value 0.744$)$ and unstable angina (15.2\% vs.

\begin{tabular}{llc}
$\begin{array}{l}\text { Table 1: Gensini number assigned according to } \\
\text { degree of luminal narrowing }\end{array}$ \\
\hline Luminal narrowing (\%) & Grading of disease & Gensini number \\
\hline $30-50$ & Mild & 01 \\
$51-70$ & Moderate & 02 \\
$71-90$ & Severe & 04 \\
$91-99$ & Subtotal occlusion & 08 \\
100 & Total occlusion & 16 \\
\hline
\end{tabular}

Table 2: Gensini multiplying factor assigned according to the location of lesion in the coronary tree

Location of lesion Multiplying factor

Left main stem

Proximal left anterior descending artery, 2.5

proximal left circumflex artery

Mid left anterior descending artery

Distal left anterior descending artery, first diagonal, mid left circumflex artery, distal left circumflex artery, obtuse marginal, proximal right coronary artery, mid right coronary artery, distal right coronary artery, posterior descending artery

Second diagonal and postero-lateral ventricular branch

Table 3: Comparison between normouricemic and hyperuricemic groups

\begin{tabular}{lccc}
\hline Parameter & $\begin{array}{c}\text { Hyperuricemic } \\
(\mathbf{n}=\mathbf{3 5})\end{array}$ & $\begin{array}{c}\text { Normouricemic } \\
(\mathbf{n}=\mathbf{4 7})\end{array}$ & P value \\
\hline Mean uric acid level & $7.59 \pm 8.12$ & $4.83 \pm 0.66$ & $<0.001$ \\
(mg/dL) & & & \\
Gensini score & $33.33 \pm 10.64$ & $22.90 \pm 6.75$ & $<0.001$ \\
Diseased vessels & $2.36 \pm 1.11$ & $2.12 \pm 0.99$ & 0.319 \\
Critical lesions & $1.03 \pm 0.84$ & $0.63 \pm 0.72$ & 0.031 \\
Total occlusions & $0.67 \pm 0.47$ & $0.37 \pm 0.48$ & 0.007 \\
Mean age (in years) & $53.76 \pm 6.28$ & $53.45 \pm 6.25$ & 0.828 \\
Male: & $21(63.6):$ & $29(59.2):$ & 0.685 \\
Female (n, \%) & $12(36.4)$ & $20(40.8)$ & \\
Family history & $13(39.4)$ & $19(38.8)$ & 0.955 \\
of CAD (\%) & & & \\
Smoker (\%) & $8(24.2)$ & $17(34.7)$ & 0.313 \\
Dyslipidemia (\%) & $21(63.6)$ & $15(30.6)$ & 0.003 \\
Hypertensive (\%) & $22(66.7)$ & $24(49.0)$ & 0.113 \\
Obesity & $17(51.5)$ & $22(44.9)$ & 0.556 \\
(BMl>30 kg/M $\left.{ }^{2}\right)$ & & & \\
Diabetes mellitus (\%) & $11(33.3)$ & $12(24.5)$ & 0.382 \\
STEMI (\%) & $19(57.6)$ & $28(57.1)$ & 0.969 \\
NSTEMI (\%) & $9(27.3)$ & $15(30.6)$ & 0.744 \\
Unstable angina (\%) & $5(15.2)$ & $6(12.2)$ & 0.705 \\
\hline
\end{tabular}

$12.2 \%, \mathrm{p}$ value 0.705$)$ except dyslipidemia (63.6\% vs. $30.6 \%$, p value 0.003$)$. Regarding angiographic severity hyperuricemic patients are associated with higher Gensini score $(33.33 \pm 10.64$ vs. $22.90 \pm 6.75$, p value $<0.001)$, frequent number of critical lesion $(1.03 \pm 0.84$ vs. $0.63 \pm 0.72$, $\mathrm{p}$ value 0.031$)$ and total occlusion ( $0.67 \pm 0.47$ vs. $0.37 \pm 0.48$, $\mathrm{p}$ value 0.007$)$; though there was no significant difference in number of involved vessels ( $2.36 \pm 1.11$ vs. $2.12 \pm 0.99$, $\mathrm{p}$ value 0.319 ) (Table 4). 


\begin{tabular}{|c|c|c|c|c|c|}
\hline \multirow{2}{*}{$\begin{array}{l}\text { Dependent variable: } \\
\text { Serum uric acid level }\end{array}$} & \multirow{2}{*}{$\begin{array}{c}\text { Standardized coefficients } \\
\text { Beta }\end{array}$} & \multirow[t]{2}{*}{$\mathbf{t}$} & \multirow[t]{2}{*}{ Sig. } & \multicolumn{2}{|c|}{$95 \%$ confidence interval for B } \\
\hline & & & & Lower bound & Upper bound \\
\hline Constant & & 8.331 & 0.000 & 2.797 & 4.552 \\
\hline Gensini score & 0.418 & 4.430 & 0.000 & 0.036 & 0.094 \\
\hline Total occlusions & 0.338 & 3.589 & 0.001 & 0.462 & 1.613 \\
\hline
\end{tabular}

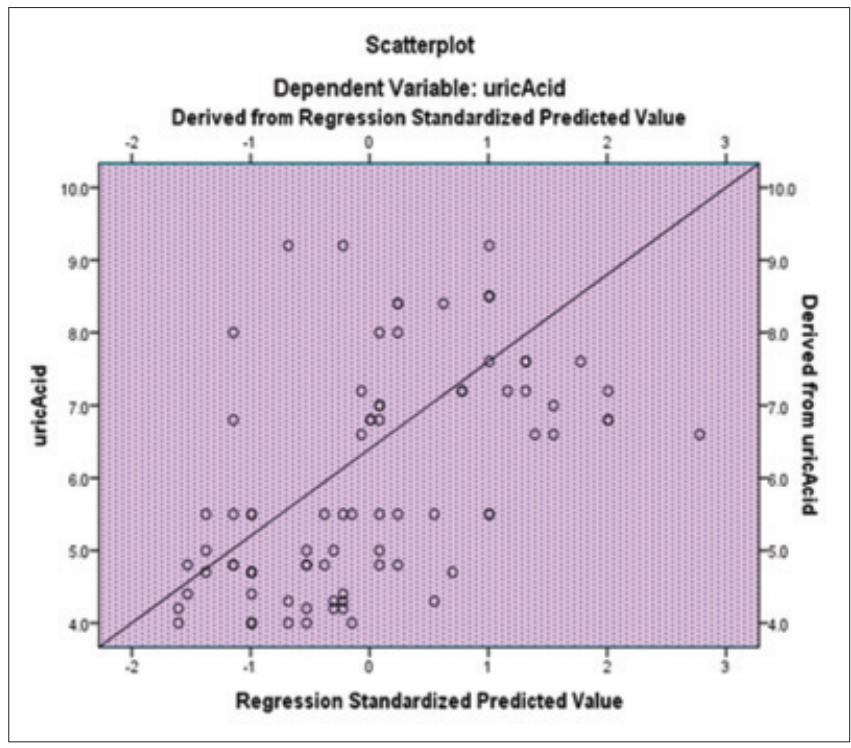

Figure 1: Correlation between serum uric acid level and Gensini score $(p<0.001)$

On regression analysis, higher serum level of uric acid was significantly correlated with higher Gensini score (beta $0.418, \mathrm{t} 4.430$, p value $<0.001,95 \%$ CI 0.036 and 0.094 ) and frequent number of total occlusion (beta 0.338, t 3.589, p value $0.001,95 \%$ CI 0.462 and 1.613) (Figure 1).

\section{DISCUSSION}

In this observational cross-sectional study we found association of hyperuricemia with the angiographic severity of CAD using Gensini scoring system. The higher serum uric acid levels was associated with higher Gensini score, frequent total occlusions and critical lesions in patients with Coronary Artery Disease compared to patients with normal uric acid level and also correlated with higher Gensini score and frequent total occlusions. Coronary angiography is the gold standard for diagnosis of CAD. Neeland IJ et al compare coronary angiographic scoring systems (Gensini) with intravascular ultrasound derived plaque burden in a CAD population undergoing angiographic evaluation and found strong correlation with each other and also with atherosclerotic plaque burden. ${ }^{6}$ Sinning $C$ et al also proposed that both Syntax scoring system and Gensini scoring system in combination with clinical variables could be used to predict the cardiovascular prognosis in CAD patients even during long-term follow-up. ${ }^{7}$ Gensini coring systems therefore appear to be a valid estimate of CAD plaque burden. ${ }^{6}$

Similar to our findings, Sinan DO et al also found association of serum uric acid level with the presence and severity of CAD. ${ }^{8}$ Azmat EQ et al also reported the association of hyperuricemia with higher Gensini score, more frequent total occlusions and critical lesions in men presenting with acute coronary syndrome. ${ }^{9}$ Duran $\mathrm{M}$ et al observed higher Gensini score, high number of diseased vessels, critical lesions, and total occlusion in hyperuricemic patients; serum uric acid level as independent predictor of multi vessel disease; and also the association of hyperuricemia with CAD severity even in non-diabetic and non-hypertensive patients. ${ }^{10}$ Xiong $\mathrm{Z}$ et al used clinical SYNTAX severity scoring system and also found significant association with the severity and complexity of CAD. ${ }^{11}$

Contrary to our result Gur M et al concluded that uric acid level is associated with the presence but not with the severity of coronary artery disease. ${ }^{12} \mathrm{Lu} \mathrm{P}$ et al observed a higher CAD severity in women having higher uric acid level, although uric acid was not independently associated with CAD or CAD severity either in women or in men. ${ }^{13}$

Ndrepepa G et al concluded that uric acid level predicts an increased risk of cardiac mortality across all subgroups of patients with CAD and the association between uric acid level and cardiac or all-cause mortality had a 'J-shaped' pattern with lowest risk of death in patients with uric acid levels between $5 \cdot 17$ and $<6.76 \mathrm{mg} / \mathrm{dL} .{ }^{14}$ Kojima $\mathrm{S}$ et al suggest that hyperuricemia after AMI is associated with the development of heart failure. ${ }^{15}$ Tamariz L et al found greater risk of atrial fibrillation in patients with elevated serum uric acid levels, particularly among blacks and women. ${ }^{16}$ Demir B et al observed a positive correlation between severity of calcific aortic stenosis and serum uric acid levels. ${ }^{17}$ A large scale prospective study is needed to determine the prognostic significance of hyperuricemia over cardiovascular events.

\section{CONCLUSION}

In conclusion, we found association of Hyperuricemia with severity of CAD. The higher serum uric acid levels 
was associated with higher Gensini score, frequent total occlusions and critical lesions in patients with Coronary Artery Disease compared to patients with normal uric acid level and also correlated with higher Gensini score and frequent total occlusions. We suggest large scale prospective study to determine prognostic significance of Hyperuricemia.

\section{REFERENCES}

1. Uday K, Sergey Z, Jennifer LF, Takahiko N, Carlos R, Wei M, et al. Hyperuricemia induces endothelial dysfunction. Kidney International 2005; 67: 1739-1742.

2. Kim SY, Guevara JP, Kim KM, Choi HK, Heitjan DF and Albert DA. Hyperuricemia and coronary heart disease: a systematic review and meta-analysis. Arthritis Care Res 2010; 62(2):170-180.

3. Clinical implication of endothelial dysfunction in patients with essential arterial hypertension and urate dysbolism with renal damage. Ter Arkh. 2011;83(10):36-40.

4. Hamaguchi S, Furumoto T, Tsuchihashi-Makaya M, Goto K, Goto D and Yokota T. JCARE-CARD Investigators. Hyperuricemia predicts adverse outcomes in patients with heart failure. Int J Cardiol 2011;151(2):143-147.

5. Rathmann W, Funkhouser E, Dyer AR, Sesti G, Perticone F and Pujia A. Relations of hyperuricemia with the various components of the insulin resistance syndrome in young black and white adults: the CARDIA study. Ann Epidemiol 1998;8(4):250-261.

6. Neeland IJ, Patel RS, Eshtehardi P, Dhawan S, McDaniel MC and Rab ST. Coronary Angiographic Scoring Systems- An Evaluation of Their Equivalence and Validity. Heart J. 2012; 164(4):547-552.

7. Sinning C, Lillpopp L, Appelbaum S, Ojeda F, Zeller T, Schnabel R, et al. Angiographic score assessment improves cardiovascular risk prediction: the clinical value of SYNTAX and Gensini application. Clin Res Cardiol 2013;102(7):495-503.
8. Deveci OS, Kabakci G, Okutucu S, Tulumen E, Aksoy H, Kaya EB, et al. The association between serum uric acid level and coronary artery disease. Int J Clin Pract 2010;64(7):900-907.

9. Qureshi $A E$, Hameed $S$ and Noeman A. Relationship of serum uric acid level and angiographic severity of coronary artery disease in male patients with acute coronary syndrome. Pak J Med Sci 2013; 29(5):1137-1141.

10. Duran M, Kalay N, Akpek M, Orscelik O, Elcik D and Ocak A. High Levels of Serum Uric Acid predict severity of ischemic heart disease in Patients with Acute Coronary Syndrome. Angiology 2012; 63(6):448-452.

11. Xiong Z, Zhu C, Qian X, Zhu J, Wu Z and Chen L. Predictors of clinical Syntax score in coronary artery disease: serum uric acid, smoking, and Framingham risk stratification. J Invasive Cardiol 2011; 23(12):501-504.

12. Gur M, Yilmaz R, Demirbag R and Aksoy N. Relation of serum uric acid levels with the presence and severity of angiographic coronary artery disease. Angiology 2008; 59(2):166-171.

13. Lu P, Hu D, Lu J, Wang W and Chen $B$. The association between uric acid and coronary heart disease. Zhonghua Nei Ke Za Zhi 2002;41(8):526-529.

14. Ndrepepa G, Braun S, King L, Fusaro M, Tada T and Cassese S. Uric acid and prognosis in angiography-proven coronary artery disease. Eur J Clin Invest 2013;43(3):256-266.

15. Kojima S, Sakamoto T, Ishihara M, Kimura K, Miyazaki S and Yamagishi M. Prognostic usefulness of serum uric acid after acute myocardial infarction (the Japanese Acute Coronary Syndrome Study). Am J Cardiol 2005; 96(4):489-495.

16. Tamariz L, Agarwal S, Soliman EZ, Chamberlain AM, Prineas $R$ and Folsom AR. Association of serum uric acid with incident atrial fibrillation (from the Atherosclerosis Risk in Communities [ARIC] study). Am J Cardiol 2011; 108(9): 1272- 1276

17. Demir B, Caglar IM, Ugurlucan M, Ozde C, Oktay Tureli H and Ciftci S. The Relationship Between Severity of Calcific Aortic Stenosis and Serum Uric Acid Levels. Angiology 2012; 63(8): 603-608. 